\title{
ENSAIO DE COMBATE AO ÁCARO DA LEPROSE DE CITROS Brevipalpus phoenicis (GEIJSKES, 1939) COM NOVO JUVENÓIDE E OUTROS ACARICIDAS
}

\author{
A.I. ClaRI; M.A.C. CARDOSO; R. IIAMAMURA; R.C. RANGEL; E.R. REgitANO; \\ L.F. MESQUITA \\ Dep. de Zoologia da ESALQ/USP, Caixa Postal, 9 - CEP: 13\$18-900-Piracicaba,SP. \\ F.A.M. MARICONI \\ Prof. Titular do Dep. de Zoologia da ESALQ/USP, Caixa Postal, 9 - CEP: 13418-900-Piracicaba,SP.
}

\begin{abstract}
RFSUMO: Foi montado um ensaio visando conhecer a eficiência do flucicloxurom (juvenóide constituído de benzoilfenil-uréia substituída), do propargite e do bromopropilato, no combate ao acaro da leprose (Brevipalpus phoenicis Geijskes, 1939). Os produtos foram empregados nas seguintes dosagens: A) testemunha; B) flucicloxurom, 10g; C) flucicloxurom, 15g; D) flucicloxurom, 20g; E) flucicloxurom, 30g; F) propargite, 72g; G) bromopropilato, 37,5g (tratamento padrão), sendo os valores supracitados quantidade de ingrediente ativo por 100 litros de calda. Foram aplicados 6 litros de calda por laranjeira, com pulverizador notorizado costal. Foram feitas 5 avaliações do combate: uma prévia (02 dias antes da pulverização) e quatro outras $(07,20,34$ e 50 dias pós-aplicação). A partir dos resultados obtidos, conclui-se que os tratamentos mais eficientes foram o propargite e o bromopropilato. Descritores: ácaro da Jeprose, Brevipalpus phoenicis, controle químico, laranjeiras, juventide.
\end{abstract}

\section{CITKUS IEPROSIS MITE CONTROL (Brevipalpus phoenicis GEIJSKES, 1939) WITII FLUCYCLOXURON, PROPARGITE ANI BROMOPROPILATE}

\begin{abstract}
Lambda$ field test was carried out on adult orange trees sprayed with flucycloxuron, propargite and bromopropilate to check their efficiency in controlling leprosis mite. Treatments used were: A) check; B) flucycloxuron, 10g; C) flucycloxuron, 15g; D) flucycloxuron, 20g; E) flucycloxuron, 30g; F) propargite, 72g; G) bromopropilate, 37,5g. Quantities indicated are grams of active ingredients per 100 liters of water. Control evaluations were made 2 days before and $7,20,34$ and 50 days after spraying. Treatments of propargite and bromopropilate turned out to be the most efficient. Treatments B, C, D, E were not efficient at all.
\end{abstract}

Key Words: citrus leprosis mite, Brevipalpus phoenicis, chemical control, citrus, juvenoid.

\section{INTRODUÇत̃̃o}

O ácaro da leprose é, no momento, a mais importante praga dos citros. Todos os anos são experimentados novos defensivos agrícolas com o objetivo de encontrar produtos com bom poder residual, mais econômicos, que menos interfiram no equilibrio biológico e que sejam menos tóxicos. OLIVEIRA et al. (1989) verificaram que a fempropatrina isolada ou misturada ao fenitrotiom mostrou-se altamente cficiente até 102 dias depois da aplicação. DESIDÉRIO et al. (1989) verificaram que os alquenóis multimetílicos (feromônios) aumentaram o período residual nas misturas feromônio + dicofol e feromônio + enxofre e em outros casos ensaiados, o período residual permaneceu inalterado. PAPA et al. (1989) comprovaram que o piretróide bifentrina foi excelente controlador do ácaro por mais de 100 dias. ALVES JOSÉ ct al. (1989) comprovaram que a fempropatrina foi excelente contra o ácaro até 60 dias da aplicação, em dois campos expcrimentais. AFFÉRRI et al. (1989) pulverizaram laranjeiras com quinometionato, flubenzimina, bromopropilato e azociclotina. Depois de alguns dias, laranjas foram colhidas e levadas para o laboratório e nelas foram colocadas fêmeas do ácaro. Observações feitas $06,14,18,42$ e 62 dias após a pulverização demonstraram que quase todos os tratamentos tem boa ação residual sobre os ácaros descendentes, com mortalidade superior a $80 \%$. A azociclotina, na maior concentração, atuou até 42 dias da aplicação. MARICONI ct al. (1989) comprovaram que o dicofol, clofentezina e pirctróide RU-1.000 foram excelentes aos 35 dias da pulverização.

O presente trabalho teve como principal objetivo verificar o efeito de quatro dosagens de nucicloxurom contra o ácaro da leprose. O flucicloxurom é juvenóide (benzoil-fenil-uréia substituída). 


\section{MATERIAL E MÉTODOS}

Iocal: Campo experimental instalado na Chácara São Luís, de propriedade do Sr. Irineu Parolina, em Piracicaba, Estado de São Paulo.

Pomar: Formado de laranjeiras da variedade "Pera", com idade aproximda de 15 anos; espaçamento de 5,40m x 4,20m (440 plantas/ha).

Tratamentos: Sete, com a testemunha, havendo quatro repetições para cada tratamento, totalizando 28 parcelas, tendo sido adotado o método de blocos ao acaso. Cada parcela era constituída de três plantas. Os tratamentos, produtos comerciais, formulações e consumo de materiais podem ser vistos na Figura 1.

Aplicação: Realizada em 28 de maio de 1989, com pulverizadores costais motorizados. Cada parcela, exceto a testemunha, recebeu 18 litros de calda (06 1/planta). Para melhor homogeneização, os aparelhos eram carregados com 9 litros de calda; em seguida, aplicava-se toda a calda de um lado das três plantas que compunham a parcela. Esta operação era repetida na outra lateral, completando-se assim os 18 litros por parcela em toda a parte aérea das laranjeiras. Foi utilizado o espalhante-adesivo "Extravon", na dosagem de 25 $\mathrm{cm}^{3}$ por 100 litros de calda.

Coletas: Foram realizadas 05 coletas: a prévia, em 26 de maio de 1989 (02 dias antes da aplicação) e quatro outras, respectivamente, em 04 e 17 de junho e 01 e 17 de julho de 1989 (portanto, após $07,20,34$ e 50 dias da aplicação). Em cada uma delas, foram apanhados 5 frutos da planta central da parcela, resultando em 20 frutos por tratamento. Eram coletados frutos não verdes e com verrugose, postos em saquinhos rotulados e levados para o laboratório logo após o término da coleta.

Contagens: Feitas em laboratório, com o auxílio de microscópios estereoscópicos, com aumento de 30 vezes. Em cada fruto eram marcadas quatro áreas circulares, feitas com vazador $\mathrm{n}^{\circ} 12(18 \mathrm{~mm}$ de diâmetro interno), sobre as regiões que apresentavam verrugose. Todas as formas ativas do ácaro da leprose foram contadas (TABELA 1 ).

Reduçāo real ou eficiência: Foi calculada pela fórmula de "Abbott", tomando-se por base a população de ácaros de cada tratamento, transformada em porcentagem de sobrevivência (TABELA 1).

Análise estatística: As populações de ácaro, em todas as contagens, em cada parcela, foram transformadas em raiz quadrada de $x+0,5$, sendo x a quantidade de ácaro em cada parcela. Os resultados foram analisados pelo teste de "Tukey" ao nível de $5 \%$ de probabilidade. $O$ coeficiente da variação também foi estimado (TABELA 2).

Ácaros predadores: Tanto na contagem prévia, como nas 4 pós-pulverização, foram contados os ácaros fitoseiídeos. A população foi muito baixa $\mathbf{e}$, às vezes, nula (TABELA 3).

\section{RESULTADOS E DISCUSSÃO}

Pelos resultados da análise estatística, a nível de $5 \%$ de probabilidade, e pelas eficiências, verifica-se:

Contagem prévia: (02 dias antes da aplicação): os tratamentos não diferiram significativamente entre si.

$1^{a}$ Contagem (07 dias após a pulverização): os tratamentos ainda não diferiram significativamente entre si, mas o melhor é $\mathrm{G}$ (bromopropilato) e em segundo lugar $F$ (propargite).

$2^{a}$ Contagem (20 dias após): embora $G$ (bromopropilato) e F (propargite) não diferissem da testemunha, foram os melhores tratamentos, com eficiências de $90,3 \%$ e $87,7 \%$, respectivamente.

$3^{2}$ Contagem (34 dias após): somente $\mathrm{G}$ diferiu estatisticamente da testemunha e sua eficiência foi de $98,3 \%$. F, embora não diferindo, obteve $96,9 \%$. Os outros tratamentos continuaram com maus resultados.

$4^{a}$ Contagem: (50 dias após): nenhum tratamento diferiu da testemunha, mas F e G continuaram os melhores $(96,1 \%$ e $94,3 \%$ de mortalidades reais).

\section{CONCLUSÕES}

Os tratamentos feitos com o flucicloxorom não tiveram boa eficiência; seus resultados foram de ruins a péssimos. Os melhores resultados foram obtidos com o bromopropilato $\mathrm{e}$ o propargite, principalmente dos 20 dias em diante. 


\begin{tabular}{|c|c|c|}
\hline TRATAMENTO & FORMULAÇÃO & FORMULAÇÃO (p/100 1 de água) (*) \\
\hline A - testemunha & $\ldots \ldots$ & 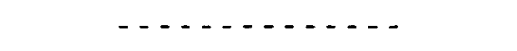 \\
\hline B - flucicloxurom & Andalin $250 \mathrm{CE}$ Uniroyal & $40 \mathrm{~cm}^{3}$ \\
\hline C - flucicloxurom & Andalin $250 \mathrm{CE}$ Uniroyal & $60 \mathrm{~cm}^{3}$ \\
\hline D - flucicloxurom & Andalin $250 \mathrm{CE}$ Uniroyal & $80 \mathrm{~cm}^{3}$ \\
\hline E - flucicloxurom & Andalin $250 \mathrm{CE}$ Uniroyal & $120 \mathrm{~cm}^{3}$ \\
\hline F - propargite & Omite $720 \mathrm{CE}$ BR & $100 \mathrm{~cm}^{3}$ \\
\hline G - bromopropilato $(* *)$ & Neoron $500 \mathrm{CE}$ & $75 \mathrm{~cm}^{3}$ \\
\hline \multicolumn{3}{|c|}{$\begin{array}{l}\text { (*) Para } 100 \text { litros: "Extravon", } 25 \mathrm{~cm}^{3} \text { e ingredientes ativos, } 10,15,20 \text { e } 30 \mathrm{~g} \mathrm{(B} \mathrm{a} \mathrm{E),} 72 \mathrm{~g} \text { (F) } \\
\text { e } 37,5 \mathrm{~g}(\mathrm{G})\end{array}$} \\
\hline
\end{tabular}

Figura 1 - Combate experimental ao "ácaro da leprose" em citros: tratamentos, formulações e consumo de material.

TABELA 1 - Combate experimental ao "ácaro da leprose" em citros: população do ácaro, a diferentes intervalos e mortalidade real (eficiência).

\begin{tabular}{cccccccccc}
\hline \hline \multirow{2}{*}{ Trata- } & \multicolumn{4}{c}{ População do Ácaro $(*)$} & \multicolumn{5}{c}{ Mortalidade Real (\%) $(* *)$} \\
\cline { 2 - 9 } mento & Prévia & $\begin{array}{c}\text { Após } \\
7 \text { dias }\end{array}$ & $\begin{array}{c}\text { Após } \\
20 \text { dias }\end{array}$ & $\begin{array}{c}\text { Após } \\
\text { 34 dias }\end{array}$ & $\begin{array}{c}\text { Após } \\
50 \text { dias }\end{array}$ & $\begin{array}{c}\text { Após } \\
7 \text { dias }\end{array}$ & $\begin{array}{c}\text { Após } \\
20 \text { dias }\end{array}$ & $\begin{array}{c}\text { Após } \\
\text { 34 dias }\end{array}$ & $\begin{array}{c}\text { Após } \\
50 \text { dias }\end{array}$ \\
\hline A & 64 & 50 & 58 & 67 & 78 & - & - & - & - \\
B & 66 & 29 & 41 & 30 & 27 & 43,8 & 31,5 & 56,5 & 66,4 \\
C & 69 & 20 & 62 & 64 & 35 & 62,9 & 0,9 & 11,4 & 58,4 \\
D & 114 & 27 & 80 & 87 & 50 & 69,7 & 22,6 & 27,1 & 64,0 \\
E & 57 & 25 & 44 & 67 & 33 & 43,9 & 14.8 & 0,0 & 52,5 \\
F & 63 & 13 & 7 & 2 & 3 & 73,6 & 87,7 & 96.9 & 96,1 \\
G & 57 & 5 & 5 & 1 & 4 & 88,8 & 90,3 & 98,3 & 94,3 \\
\hline \hline
\end{tabular}

(*) Soma das 4 parcelas.

(**) Pela fórmula transformada de Abbott:

$$
R R=\frac{\% \text { sobrev.test. }-\% \text { sobrev.trat. }}{\% \text { sobrev.test. }} \times 100
$$


TABELA 2 - Combate experimental ao "ácaro da leprose" em citros: médias da população do ácaro, a diferentes intervalos, transformadas em raiz quadrada de $x+0,5$ e resultados estatísticos (Tukey, 5\%).

\begin{tabular}{|c|c|c|c|c|c|}
\hline \multirow{2}{*}{$\begin{array}{l}\text { TRATA- } \\
\text { MENTO }\end{array}$} & \multicolumn{2}{|c|}{ M É D I A S } & POPULA Ç ÃO & \multicolumn{2}{|c|}{ DE ÁCARO } \\
\hline & Prévia & Após 7 dias & Após 20 dias & Após 34 dias & Após 50 dias \\
\hline A & $3,42 \mathrm{a}$ & $3,45 \mathrm{a}$ & 3,77 a b c & $4,11 \mathrm{ab}$ & $3,95 \mathrm{a}$ \\
\hline B & 4,01 a & $2,57 \mathrm{a}$ & $3,13 \mathrm{a} \mathrm{b} \mathrm{c}$ & $2,34 \mathrm{a} \mathrm{b} \mathrm{c}$ & $2,47 \mathrm{a}$ \\
\hline $\mathrm{C}$ & $4,03 a$ & $1,97 \mathrm{a}$ & $3,97 \mathrm{ab}$ & $3,40 \mathrm{a} \mathrm{b} \mathrm{c}$ & $3,00 \mathrm{a}$ \\
\hline $\mathrm{D}$ & $4,43 \mathrm{a}$ & $2,65 \mathrm{a}$ & $4,27 \mathrm{a}$ & $4,54 a$ & $3,37 \mathrm{a}$ \\
\hline E & $3,83 \mathrm{a}$ & $2,50 \mathrm{a}$ & $3,11 \mathrm{a} \mathrm{b} \mathrm{c}$ & 3,99 a b c & $2,77 \mathrm{a}$ \\
\hline F & 3,95 a & $1,87 \mathrm{a}$ & $1,44 \quad b c$ & 0,93 b c & $1,06 \mathrm{a}$ \\
\hline G & 3,71 a & $1,19 \mathrm{a}$ & $1,19 \quad \mathrm{c}$ & 0,84 & $1,13 \mathrm{a}$ \\
\hline C.V. (\%) & 51,28 & 44,44 & 39,13 & 49,54 & 51,75 \\
\hline D.M.S. & 4,68 & 2,40 & 2,72 & 3,33 & 3,06 \\
\hline
\end{tabular}

Médias seguidas da mesma letra não diferem estatisticamente entre si.

TABELA 3 - Combate experimental ao "ácaro da leprose" em citros: população do ácaro predador a diferentes intervalos.

\begin{tabular}{ccccccc}
\hline \hline \multirow{2}{*}{ TRATA- } & \multicolumn{2}{c}{ P O P U L A ÇA O } & D O & Á C A R O & \multicolumn{2}{c}{ P R E D A D O R } \\
\cline { 2 - 6 } MENTO & Prévia & Após 7 dias & Após 20 dias & Após 34 dias & Após 50 dias \\
\hline A & 1 & 0 & 0 & 0 & 1 \\
B & 7 & 0 & 1 & 0 & 0 \\
C & 3 & 0 & 3 & 1 & 2 \\
D & 2 & 0 & 0 & 3 & 0 \\
E & 0 & 0 & 0 & 2 & 1 \\
F & 4 & 0 & 0 & 0 & 0 \\
G & 6 & 0 & 1 & 0 & 0 \\
\hline \hline
\end{tabular}




\section{REFERENCIAS BIBLIOGRÁFICAS}

AFFERRI, F.S.; NOGUEIRA, C.E.; FAVORETO, A.J.; CHIAVEGATO, L.G. Ensaio visando o controle de Brevipalpus phoenicis (Geijskes, 1939) (Acari: Tenuipalpidae) em citros através do efeito residual de alguns acaricidas e ovicidas. In: CONGRESSO BRASILEIRO DE ENTOMOLOGIA, 12., 1989, Belo Horizonte. Resumos... Belo Horizonte : SEB, 1989. v.2, p.374.

DESIDERIO, N.D.; MATTOS, J.B.S.; DODO, H. Influência da adiçāo do feromónio Stirrup $\mathrm{M}$, a acaricidas no controle de Brevipalpus phoenicis (Geijskes, 1939) (Acari: Tenuipalpidae) em citros. In: CONGRESSO BRASILEIRO DE ENTOMOLOGIA, 12., 1989, Belo Horizonte. Resumos... Belo Horizonte : SEB, 1989. v.2, p.265.

JOSÉ, L.A.A.; LEÇA, P.F ; NAKANO, O. Controle do ácaro da leprose Brevipalpus phoenicis (Geijskes, 1939) (Acari: Tenuipalpidae) com o novo acaricida fempropatrina em duas regıōes citrícolas do Estado de São Paulo. In: CONGRESSO BRASILEIRO DE ENTOMOLOGIA, 12, 1989, Belo Horizonte. Resumos.. Belo Horizonte : SEB, 1989. v.2, p.326.
MARICONI, F.A.M.; RANGEL, R.C.; HAMAMURA, R.; CLARI, A.I.; MESQUTTA, L.F.; CARDOSO, M.A.C.; REGITANO, E.B. Ácaro da leprose Brevipalpus phoenicis (Geijskes, 1939): combate experimental em laranjeiras. Anais da Escola Superior de Agricultura "Luiz de Queiroz", Piracicaba, v.46, pt.2, p.473-483, 1989.

OLIVEIRA, W.P.; FUDO, C.H.; NAKANO, 0 . Comparaçōes de novos acaricidas com os padrōes visando o controle do ácaro da leprose Brevipalpus phoentcis (Geijskes, 1939) (Acari: Tenuipalpidae) em citros. In: CONGRESSO BRASILEIRO DE ENTOMOLOGIA, 12, 1989, Belo Horizonte. Resumos... Belo Horizonte : SEB, 1989. v.2, p.264.

PAPA, G.; ORII, F.N.; NAKANO, O. Efeito de várias formulaçōes de bifenthrin no controle do ácaro da leprose Brevipalpus phoenicis (Geijskes, 1939) (Acari: Tenuipalpidae) em citros. In. CONGRESSO BRASILEIRO DE ENTOMOLOGIA, 12., 1989, Belo Horizonte. Resumos... Bilo Horizonte : SEB, 1989. v.2, p.276.

Trabalho entregue para publicação em 08.11.90

Trabalho aprovado para puhlıcação em 30.09.92 